\title{
ITFComp: A Compression Algorithm for ARM Architecture Instruction Trace Files
}

\author{
Mohammad Qasem \\ Fraunhofer, FKIE \\ Fraunhoferstr. 20 \\ 53343 Wachtberg, Germany \\ mohammad.qasem@fkie.fraunhofer.de
}

\author{
Lukas Pustina \\ CenterDevice $\mathrm{GmbH}$ \\ Rheinwerkallee 3 \\ 53227 Bonn, Germany \\ lukas.pustina@centerdevice.com
}

\begin{abstract}
Testing the performance of a new computational component is costly due to the need of prototyping different setups. Therefore, trace driven hardware simulations are used. Instruction Trace Files (ITFs) are files containing traces of executed instructions in a program's run and are used as an input for hardware simulations. ITFs tend to be large in size, causing a storage challenge. Many trace reduction techniques exist to deal with the ITFs' storage challenge. In this paper we introduce ITFComp, a compression algorithm that combines general purpose compression methods with knowledge about ARM architecture ITFs' structure to reduce their size. ITFComp also works on compressing data memory addresses accessed by instructions within ITFs to further reduce an ITF size. Results show a reduction of 600 times on average when combined with LZMA compression algorithm. This reduction is 4 times better than when using LZMA alone, and 10 times better than when using DEFLATE. ITFComp introduces a negligible overhead in the decompression time (less than $1 \%$ ).
\end{abstract}

\section{Keywords}

Performance, Instruction Trace Files, Compression, Memory Addresses, Hardware, Simulation, ARM, Architecture

\section{INTRODUCTION}

In the recent years, there has been a wide spread of handheld computational devices, one of such devices is the smartphone. In the smartphone market, by 2014 , more than $95 \%$ of used processors were of ARM architecture [11]. When a new smartphone design is presented, its performance has to be tested. This process requires prototyping the design, and changing the prototype whenever a design change is suggested. Such prototyping is costly in time and resources.

An alternative approach to prototyping is hardware simulations [3]. Hardware simulations are done by designing a software component that simulates a hardware component such as a CPU. The performance of the simulated hardware components is tested by executing instructions on them.
Instructions are recorded from an actual benchmark run on a similar hardware. Low level executed instructions of benchmarks are recorded in files called Instruction Trace Files (ITF) in a process called tracing. ITFs are used as an input for hardware simulations.

Modern processing units with clock speeds of few gigahertz execute billions of instructions per second. Therefore, recording the instructions of a program that lasts few seconds in execution would generate a very large ITF causing a storage challenge. Another factor that contributes to the storage challenge is the need most of the times to store multiple runs of the same benchmark with different inputs. This process creates multiple large ITFs per benchmark.

In this paper, we focus on presenting a solution for the ARM based ITFs storage challenge. ARM architecture was chosen due to its wide spread as an architecture in smartphones and handheld computational devices. The target ITFs are text files where each line represents an executed instruction during tracing. An executed instruction consist of an instruction address, the instruction, the parameters, and any optional flags or data memory accesses.

General purpose compression algorithms can be used to compress ITFs. However, such algorithms lack knowledge of ITFs structure that could be used to further reduce an ITF size. Such knowledge also allows for being more flexible while decompressing ITFs by allowing for random access within the compressed files.

This knowledge comes from the fact that an ITF consists of repeating instruction segments called basic blocks with unique entry point and exit point. Instructions in repeating instances of the same basic block differ in execution flags, and data memory accesses of load and store instructions. Therefore, an ITF can be represented as a sequence of basic blocks identified by their entry points, instead of having to store instructions line by line. Differences between two instances of the same basic block can be stored separately.

In this paper, a compression algorithm for ARM based ITFs called ITFComp is introduced. This algorithm makes use of ITF structure mentioned earlier and combines it with general purpose compression methods to reduce ITFs storage requirements. ITFComp reduces the size of ITFs more than general purpose compression algorithms, and it introduces a negligible (less than $1 \%$ ) overhead on the decompression time.

ITFComp further reduces an ITF size by compressing data memory addresses accessed by the instructions within an ITF. ITFComp makes use of the locality and the access patterns that instructions usually follow when accessing the data memory [7].

In summary, ITFs are used in many performance related stud- 
ies, one of which is hardware simulations and testing. Hardware simulations saves time and money compared to prototyping different designs. ITFs tend to be large in size requiring compression. General purpose compression algorithms lack domain specific knowledge about ITFs structure. This knowledge could be used to further reduce ITFs size. In this paper, we suggest a new compression algorithm called ITFComp that makes use of such knowledge.

The remainder of this paper is as follows, the following section is an overview of the covered related work. An algorithm overview is given in the third section. The fourth section discuss the first step of the algorithm: compressing the sequence of instructions in an ITF. The fifth section discusses the second step: compressing data memory addresses accessed in an ITF. The sixth section shows the used evaluation metrics and the results of evaluating the suggested algorithm. The last section is a summary.

\section{RELATED WORK}

Many compression algorithms that are ITF specific exist. Some algorithms work on instruction traces, while others target the data memory access traces. The presented ITFComp algorithm works on both instructions and memory addresses. Therefore, ITF specific compression algorithms of different targets were overviewed. None of the found and reviewed algorithms deals with ARM text trace files as with ITFComp.

In this section, a summary of overviewed ITF specific compression algortihms is given. The overview is given in two subsections: Instruction traces compression algorithms, and memory addresses compression algorithms.

\subsection{Instruction Traces Compression Algorithms}

For instructions compression, trace filtering was used in [8]. It is done by discarding parts of the ITF that have low impact on the simulation. This depends on which component in the target hardware is being evaluated.

A compression method was proposed in [1] that works by storing a map between instruction addresses and instruction words. Once this is done, only addresses of instructions need to be stored in an ITF rather than the whole instruction. Following that, addresses are compressed by dividing them into sets of sequential and non-sequential groups of addresses. Each type of sets is compressed in a different way.

In [7], a compression method called PDI was introduced. The algorithm represents an extension for PDATS [6] memory trace compression algorithm and works in two steps: The first step scans an ITF looking for the most common 256 instruction words. The words are stored in a dictionary that maps values of 1-256 to the instruction words. Common instructions are replaced with a value of 1-256 corresponding to their dictionary index, while the rest are kept as they are. The second step is applied in a manner similar to PDATS, which is explained in the following subsection 2.2.

A compression algorithm that makes use of streams in ITFs was introduced in [9]. An instruction stream is a series of instructions that are executed sequentially until a flow changing instruction. Streams are different from basic blocks for not having a unique entry and exit points. Therefore, some instructions might show in multiple streams. The algorithm detects instruction streams and stores the most common ones in a dictionary. Occurrences of common streams are replaced with their dictionary index.

Hardware based real time compression of ITFs was intro- duced in [10]. The work is done by compressing instructions while tracing them in real time. The compression is done in a manner similar to the work in [9].

\subsection{Memory Addresses Compression Algorithms}

For memory addresses compression, two approaches were suggested in [15]. Both approaches work by sampling cache hits, either in a timely manner or depending on their level in the cache memory. Both approaches introduce a small error rate in simulations.

PDATS [6] is a memory addresses compression algorithm. PDATS work on extracting the distances between data memory addresses, then applies run length encoding to compress them. This approach is lossless.

Another lossless compression scheme was suggested in [13]. The algorithm applies general purpose compression on the distances between stored addresses in a memory trace file rather than the addresses themselves. Therefore, the algorithm represents a generalization of PDATS mentioned earlier.

As mentioned earlier, none of the found and reviewed methods deals with ARM ITFs that are text based. Text based ARM ITFs show instructions addresses, instructions, and their parameters.

\section{ALGORITHM OVERVIEW}

ITFComp increases the reduction in size of ITFs when applying general purpose compression. It achieves that by using knowledge about ITFs structure. As stated in the introduction, this knowledge is represented in basic blocks being the building components of an ITF, and memory access addresses locality and patterns. ITFComp uses this knowledge to compress an ITF in two steps, making it a multi-pass compression algorithm: compressing instructions after extracting data memory addresses, and compressing extracted memory addresses.

A basic block is a series of instructions that has a single unique entry point and a single unique exit point. Static basic blocks is a term used to define high level language blocks such as the body of a for loop. In an ITF, dynamic basic blocks exist. Dynamic basic blocks represent an execution of a static basic block or a part of it due to interrupts or flow changing instructions. In this paper, the term basic block is used for a dynamic basic block unless mentioned otherwise.

A basic block might show multiple times within an ITF, creating different instances of the basic block. Instances of a basic block might differ in execution flags, or data memory addresses accessed by instructions within.

A segment of an ITF is shown in Listing 1 . The segment contains two consecutive instances of the same basic block starting with the address 0x10001014, and a third different basic block with the address $0 \times 1000103 \mathrm{c}$. The two instances of basic block $0 \times 10001014$ differ in data memory addresses and flags as can be seen in the instructions $0 \times 10001024$, and $0 \times 10001038$ respectively.

Data memory addresses accessed by the same instruction usually exhibit locality among the different instances of its basic block. Accessed addresses follow certain patterns that could be used to allow for a high amount of reduction in size. For example, storing the distances between successive addresses rather than absolute addresses [13][6], reduces the amount of space required for storage per memory access. An example of a pattern that exist within memory accesses is a constant location in memory in the case where an instruction reads and writes to a fixed variable in the high level language. Another 


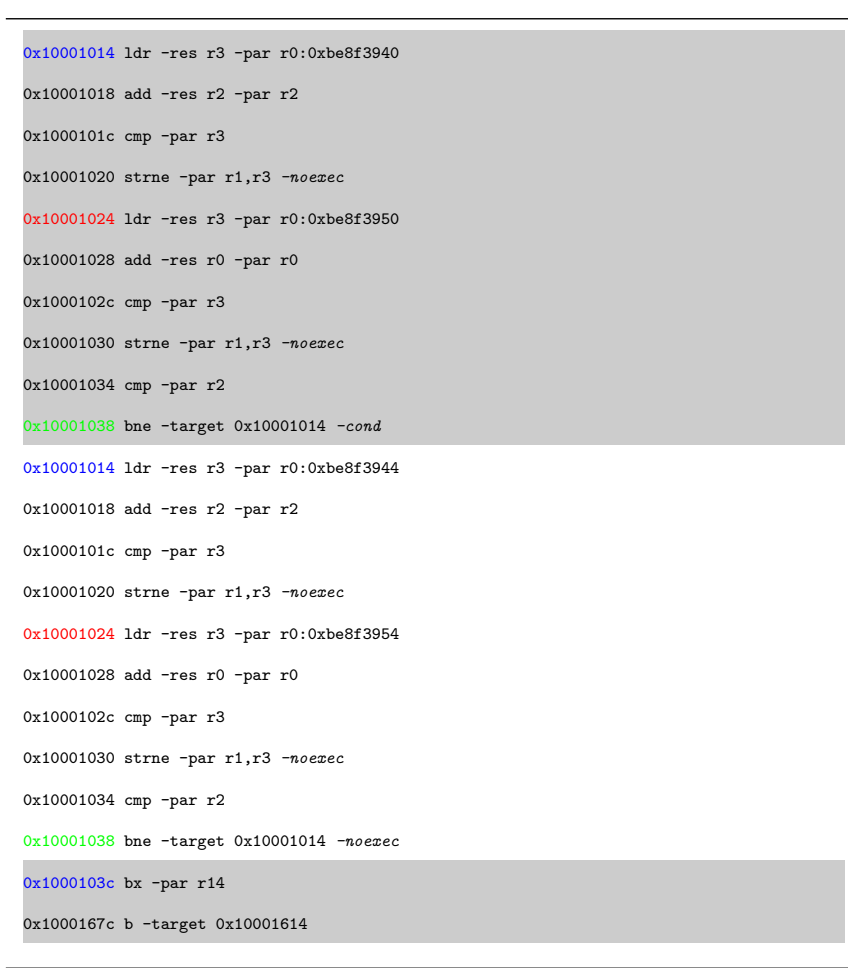

Listing 1: A segment of an ITF showing a repeating basic block, followed by a different basic block

example is having a periodic access pattern when reading from a list like structure sequentially.

ITFComp compression works by generating a list of basic blocks within an ITF by identifying their unique entry and exit points. It then proceeds by representing the ITF as a sequence of basic blocks identified by their unique entry points. One instance per basic block is stored as a template into a dictionary. A template is a basic block instance with data memory addresses and flags replaced with place holders.

Instructions are identified by their relative address in their basic block. For example, in Listing 1, the highlighted instruction $0 \times 10001024$ is identified as instruction number 16 (hex 10$)$ in basic block 0x10001014. Data memory addresses are stored per instruction as the first accessed address by the instruction, and a list of distances to each consecutive access of that instruction. Flags are stored within the basic blocks sequence of a compressed ITF in different sets. A flag set contains instruction numbers that has its flag within the next basic block.

The decompression of an ITF goes by reading the sequence of basic blocks. For each element, the stored basic block template from the dictionary is written. Missing data memory addresses and flags are added depending on the instance number.

The general sketch of the ITFComp algorithm mentioned in this section still lacks information such as data memory addresses compression, and decompression processes details. Extracting basic blocks and their sequence in an ITF is explained in the next section, while dealing with data memory addresses compression is detailed in Section 5.

\section{INSTRUCTIONS COMPRESSION}

As explained in the previous section, the ITFComp algorithm compresses an ITF in two steps: compressing instructions of the ITF, and compressing data memory addresses. In this section the first step is explained in details. The use of the result of this step during the decompression of an ITF is also detailed.

ITFComp detects the sequence of basic blocks executed in an ITF. It replaces them with their unique entry address resulting in a compressed file. For each basic block, one instance with data memory addresses and flags replaced with place holders to be filled during the decompression process is stored as a template.

The result of the aforementioned process is a sequence of basic block unique entry addresses, with each having an instance number shown as an index on the address. Flags are stored into sets in the resulting basic blocks sequence, per basic block. The sets contain the instruction numbers for which the flag should be added in the template during decompression. An example of such sequence is shown in Table 1 under the "Sequence" column.

As mentioned in the previous section, data memory addresses are extracted and stored per instruction. Instructions are identified by their relative locations within their basic block. Extracted data memory addresses compression is explained in the following section.

Table 1 is an example showing the result of compressing a segment of an ITF. The input segment used is shown in Listing 1 and consists of three basic blocks; two instances of the basic block 0x10001014 and one instance of 0x1000103c. The table contains the three elements resulting of the ITFComp algorithm; the sequence of basic blocks and the flag sets in the first column, the dictionary of templates in the second column, and extracted data memory addresses for each memory access instruction in the third column. One can notice that the more instances there is of a basic block, the higher reduction in size is achieved.

Interrupts are another issue to consider. Interrupts causes a change in the flow of execution during a basic block instance execution. Therefore, they cause a basic block execution during another. Interrupts of interrupts are also possible causing a hierarchy of basic blocks within basic blocks. ITFComp deals with this by identifying the basic block in the sequence as either a base basic block, or an interrupt basic block. An identifier is added to basic blocks in the sequence as a power of 1 or 2; 1 means it is a base basic block, and 2 means it is an interrupt basic block. Interrupt basic blocks are followed by the instruction number at which the interrupt occurs.

An example sequence would be if an interrupt occurs after the 4th instruction of the second instance of basic block $0 \times 10001014$ in Listing 1 . The interrupt starting address is assumed Oxffffos. The resulting sequence of basic blocks would be: $0 x 10001014_{1}^{1}, 0 x f f f f 08_{1}^{2}(5), 0 x 10001014_{2}^{1}, 0 x 1000103 c_{1}^{1}$.

Flag sets are used per base basic block. The sets account for their base basic block and any interrupt basic block within it.

The decompression process is done by going over the sequence of basic blocks. Therefore, sets of flags, and interrupt basic blocks will be encountered before their base basic block. Once a base basic block is encountered, its template is read. For each instruction read from the template, its data memory address access is added if applicable. The flag sets and interrupt basic blocks locations are checked for possible additions after the read instruction.

If an interrupt basic block is encountered, its template is read and dealt with as a base basic block. Each of its instructions could have flags, memory addresses, or be interrupted. 


\begin{tabular}{|c|c|c|}
\hline Sequence & Elements & Data Memory Addresses \\
\hline \multirow[t]{2}{*}{$\begin{array}{l}\text { noexec }=[12,28], \\
\text { cond }=[36], 0 x 10001014_{1} \text {, } \\
\text { noexec }=[12,28,36], \\
0 x 10001014_{2}, \\
0 x 1000103 c_{1}\end{array}$} & 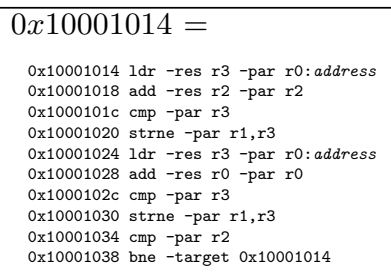 & $\begin{array}{l}0 x 10001014(0)= \\
\text { 0xbe8f3940,0xbe8f3940 } \\
0 x 10001014(16)= \\
\text { 0xbe8f3950,0xbe8f3954 }\end{array}$ \\
\hline & $\begin{array}{l}0 x 1000103 c= \\
\text { ox1000103c bx }- \text { par r14 } \\
\text { ox1000167c b }- \text { target } 0 \times 10001614\end{array}$ & \\
\hline
\end{tabular}

Table 1: The compressed sequence of the ITF segment in Listing 1

Once the base basic block is complete, the flag sets and interrupt basic blocks locations are reset. The decompression continues in the sequence and begins to generate instructions when the next base basic block is encountered.

Compressed data memory addresses are dealt with by decompressing the data memory address of an instruction depending on the instance number of the basic block read. Details of data memory addresses compression are in the next section.

\section{MEMORY ADDRESSES COMPRESSION}

Memory addresses are compressed per instruction in the ITF. In this section, the requirements for data memory addresses compression for ITFComp are given, the different tested techniques for compressing data memory addresses are shown, and their functionality explained in the compression and the decompression steps.

Instructions are identified by their relative address in a basic block. The target ITFs are of ARM architecture; a RISK architecture of a fixed 4 bytes instruction length (32-bit). Therefore, instructions are identified by numbers of multiples of 4 , following the unique entry address of their basic block. This allows for storing instructions access addresses in a single data structure (e.g dictionary) that is identified by their basic block unique entry address.

Addresses accessed by an instruction follow patterns that can be made use of. Such patterns include having a constant location to access in the memory or a periodic access over a list like structure. This introduces the first requirement for data memory addresses compression which is to make use of such underlying knowledge of their access patterns.

During the decompression of the ITF, the required accessed address of an instruction in an instance of its basic block is to be provided. This adds the requirement of random access within the compressed data memory addresses. Random access is not usually provided by general purpose compression algorithms [2].

Data memory addresses are extracted during the compression process and stored per instruction. The addresses are extracted as a set of the first data memory address, and the distance to the following address until the last instance in the ITF. Storing distances allows for storing the addresses in lower space making use of locality [6]. It also allows for detecting simple patterns. Figure 1 shows an example where distances could be used to store data memory addresses as a constant value of four instead of a linear function. Addresses can be recovered by adding an extracted distance to the previously generated address, starting from the first address, which is the first element in the set.
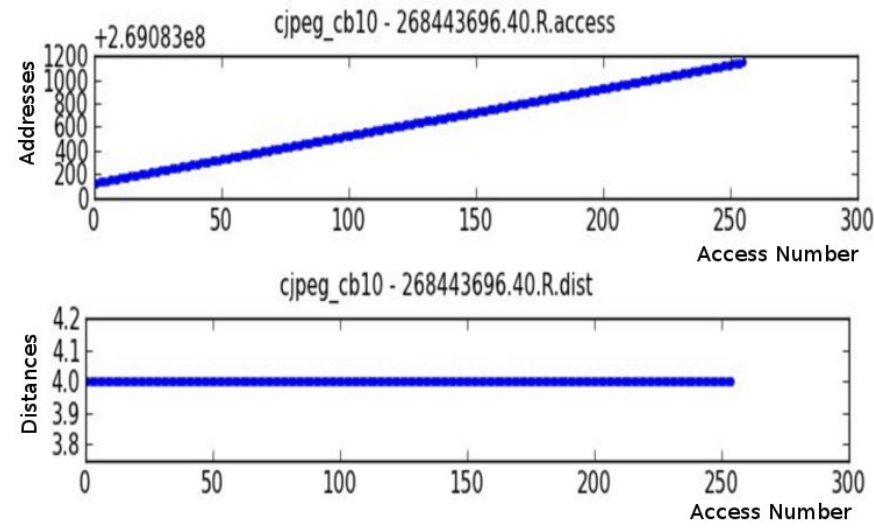

Figure 1: Data memory addresses accessed by an instruction in a cjpeg algorithm run: Absolute (above), and distances (below).

Since the application of the targeted ITFs is doing performance analysis on simulated hardware. Correctness of data memory addresses in the compressed ITF is not necessary. This allows for exploring lossy compression methods for data memory addresses, as long as the performance of memory accesses in terms of cache misses and hits does not differ to a certain level [15].

Many algorithms have been tried that comply with the requirements of using knowledge of data memory addresses structure, random access, and some being lossy. The tested algorithms were raw storage, periodicity detection, distribution compression, SEQUITUR, and patterns detection. Following is a summary of the used methods:

\section{Raw Storage}

This method stores distances between instructions addresses as they are without any compression. The goal of this method is to evaluate other compression methods according to the metrics defined in Section 6. 


\section{Periodicity Detection}

Compression: This method works by detecting periodic patterns in the distances of data memory addresses. It has been noticed that a periodic pattern is quite common in the analyzed ITFs. This is explained by having many instructions that access a fixed variable, or access a list like data container, both of which are periodic access patterns. The used algorithm to detect periodicity was a brute force algorithm that extends from 1 element to half of the input data. In each step assuming a period, and verifying it. The algorithm has a complexity $O(n)$, and a reduction of size equal to the period length over the length of the input data set.

Decompression: The required address of an instruction in its basic block instance number $N$ is retrieved by calculating $N$ modulo the period length. The element indexed by the result in the stored period is the required distance. Therefore, the decompression is $\mathrm{O}(1)$, allows for random access, and is not lossy.

\section{Distribution Detection}

Compression: This method works by representing the distances of memory addresses as a distribution. The distribution used is the histogram of the distances. The values of the histogram are chosen to guarantee a minimum of $50 \%$ reduction in size. This is done by using at $\max , 25 \%$ of the total number of input elements as histogram values. The decision is done based on the number of unique elements, if less than the threshold, they are used. Otherwise, a uniform distribution with the threshold elements count is used. $25 \%$ was chosen as a threshold as it guarantees a good size reduction, and does not cause a large loss in the resulting addresses. Thresholds of $10 \%, 15 \%, 35 \%$ were evaluated as well.

Decompression: The decompression process goes by sampling a point from the distribution. Therefore, it has an $\mathrm{O}(1)$ run time, allows for random access, and is lossy.

\section{SEQUITUR}

Compression: The SEQUITUR [4] algorithm is used to infer a context free grammar on memory addresses distances.

Decompression: The decompression process is linear. The base rule of the resulting grammar is scanned, each character size is known, and if the terminal character (the required distance) falls within/is one of the characters in the base rule, the character is expanded/chosen as the required distance. This gives the algorithm an access time of $\mathrm{O}(\mathrm{n})$. However, having information, about the number of terminal characters within a non-terminal one, allows for faster navigation within the base rule.

\section{Patterns Detection}

Compression: This algorithm represents an extension to the periodicity detection algorithm. The algorithm tries to detect less frequent patterns. The patterns are periodic with initialization, periodic with termination, and noisy periodic. A modified version of the linear search algorithm for periodicities was used to detect the first two types, and Autocorrelation was used to detect noisy periodic data. The algorithm complexity is $\mathrm{O}(\mathrm{n} \log (\mathrm{n}))$ due to using Autocorrelation.

Decompression: This method allows for more compression on the aperiodic memory access addresses. However, it adds lossiness in the case of noisy periodic data. It allows for random access within the compressed data, and has an $\mathrm{O}(1)$ access time for extracting addresses.

Table 2 shows a comparison between the different tested memory addresses compression methods in terms of being lossy, allowing for random access, and complexity.

During the evaluation of the used algorithms, due to their simplicity and good results, the periodicity detection and the patterns detection algorithms were used as bases for the other compression methods. When testing an algorithm, periodicity detection or pattern detection were applied first to compress data memory addresses, and in the case of no detection, the target algorithm is used. Therefore, the algorithms SEQUITUR, and distribution detection were applied on aperiodic data memory addresses in the presented evaluation results. In the next section, the evaluation of ITFComp, and a comparison with general purpose compression methods is shown.

\section{EVALUATION}

For evaluation, three metrics were defined and used to evaluate and compare ITFComp algorithm with general purpose compression methods. In this section, the metrics are defined, and the evaluation mechanism and results are shown.

General purpose compression methods were used as a reference, as to the best of our knowledge, no previous work dealt with text based ARM ITFs compression. The covered work in the introduction deals with ITFs containing instruction words or data memory addresses.

The used metrics are mentioned below. Detailed definition of each metric is shown at their respective results sections:

1. Compression ratio: The metric measures the amount of reduction in size when a compression method is applied.

2. Simulated run time of the ITF: The metric measures the acceptability of a resulting ITF when decompressed.

3. Wall clock simulation time: The time required to decompress an ITF and simulate it.

For these metrics, a simulator developed in [12] was used to simulate the compressed ITFs on an ARM9 architecture. The ITFs used in the evaluation process all belong to the MiBench [5] benchmark suit for ARM architecture. For every benchmark used, multiple ITFs generated executing that benchmark with different inputs were used. The evaluated benchmarks are: cjpeg, adpcm_c, adpcm_d, dijkstra, mad, crc, and blowfish_e.

Following are the results of evaluating each metric.

\subsection{Compression Ratio}

This is a measure of the reduction in size in the ITF. Therefore, it is a measure of the efficiency of the compression method. This metric was used to evaluate ITFComp when using different data memory compression methods, and to compare ITFComp to general purpose and domain specific compression methods.

The compression ratio is calculated as the size of the resulting compressed ITF over the size of the input uncompressed ITF. The lower the compression ratio is, the more size reduction on the input file there is.

In Figure 2, the results of applying ITFComp on the evaluation set are shown. The figure shows the results of using different data memory addresses compression techniques. The results show that patterns detection combined with distribution detection achieve the lowest compression ratios on average. Therefore, the highest reduction in size. This shows that 


\begin{tabular}{|l|l|l|l|l|}
\hline Algorithm & Random Read & Lossiness & Compression $(\mathrm{O})$ & Extraction (O) \\
\hline Periodicity Detection & Yes & no & $\mathrm{O}(\mathrm{n})$ & $\mathrm{O}(1)$ \\
\hline Distribution Detection & Yes & Yes & $\mathrm{O}(\mathrm{n})$ & $\mathrm{O}(1)$ \\
\hline SEQUITUR & No & No & $\mathrm{O}(\mathrm{n})$ & $\mathrm{O}(\mathrm{n})$ \\
\hline Patterns Detection & Yes & Yes & $\mathrm{O}(\mathrm{n} \log (\mathrm{n}))$ & $\mathrm{O}(1)$ \\
\hline
\end{tabular}

Table 2: A comparison of the different tested compression methods for data memory addresses

there exist a high amount of patterns within data memory addresses for the instructions in the evaluation set. It also shows that the distribution compression allows for a high compression ratio for random data.

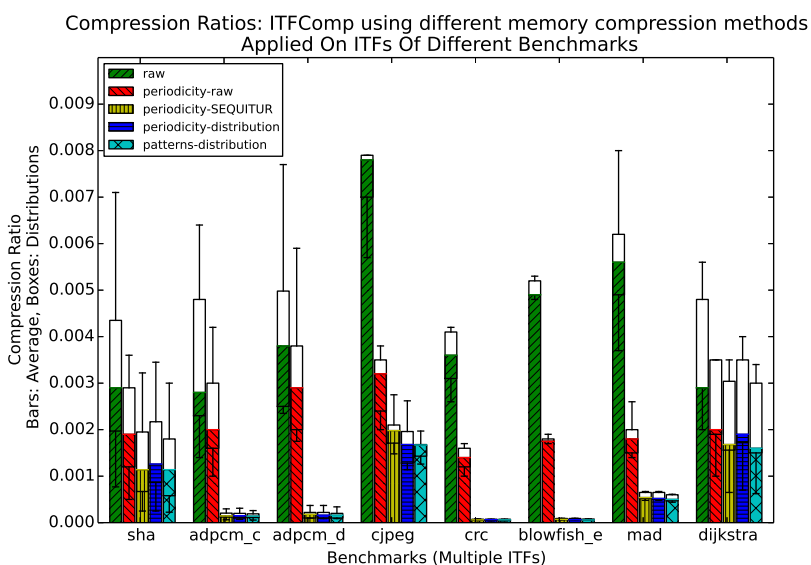

Figure 2: The compression ratio of ITFComp using different data memory addresses compression methods. Multiple ITFs of different benchmarks were used as an input.

Figure 3 shows a comparison between three general purpose compression methods: DEFLATE(gz implementation) [2], bzip2 [14], and LZMA(xz implementation) [16], when applied on the evaluation set. The figure shows that $\operatorname{LZMA}(x z)$ algorithm achieves the lowest compression ratio, and therefore the highest reduction in size. For this, LZMA is used to compare the proposed ITFComp with.

Figure 4 shows a comparison between $\operatorname{LZMA}(x z)$ compression method and ITFComp (using periodicity detection for data memory addresses compression and combined with LZMA compression). The figure shows that for the evaluation set, ITFComp managed to achieve a better average compression ratio than LZMA. The enhancement was up to 4 times in the case of adpcm for example.

ITFComp is also compared with the stream cache compression algorithm (SC.I and SC.I+Ntup when combined with Ntuple) presented in [10]. The comparison is done on ITFs from the algorithms: cjpeg, mad, sha, and blowfish_e. Table 3 shows the compression ratios achieved by ITFComp (periodicity-raw and periodicity-SEQUITUR memory addresses compression), compared to stream cache compression algorithm.

Table 3 shows that ITFComp achieves a better compression ratio for the used ITFs. When compared to SC.I, ITFComp(perio raw) achieves 6 times better compression on average. The gain goes up to 625 times better for ITFComp(periodicitySEQUITUR) for the blowfish_e input. The results of SC.I+Ntup are better than those of SC.I. However, they still fall behind by

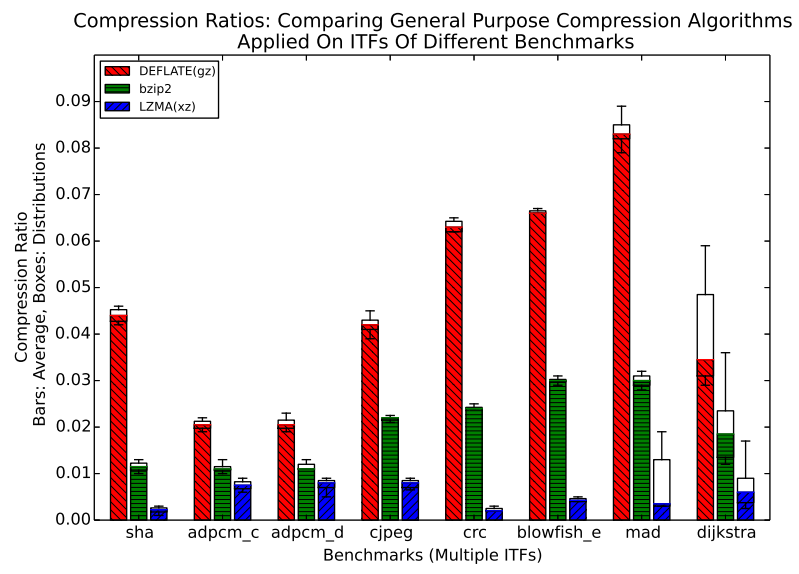

Figure 3: The compression ratio of general puprose compression methods. Multiple ITFs of different benchmarks were used as an input.

2 times on average when compared to ITFComp(periodicityraw).

In summary, ITFComp achieves the highest compression ratio when using patterns detection combined with distribution compression on data memory addresses. When compared to general purpose compression methods, ITFComp manages to achieve better compression ratios than the tested three general purpose compression methods. This shows that general purpose compression methods could use knowledge about ITFs to further reduce their size as this paper suggests. When compared to the domain specific stream cache compression method, ITFComp achieves better compression ratios. The compression ratio is 625 times better for some inputs.

\subsection{Simulated Run Time}

This is a measure of the correctness of the compressed ITFs. If the simulated run time of the compressed ITF matches that of the original ITF, it means that the used data memory addresses compression had no effect on the performance characteristics of the simulated instructions. Otherwise, a change of the simulated run time would be allowed within an acceptable experimental threshold, given that the gain in the size reduction justifies it.

Two data memory addresses compression methods have lossy compression: patterns detection, and distribution detection. The amount of lossiness is reduced when combined with periodicity compression as explained earlier. Figure 5 shows the itysimulated run time for compressed ITFs in the simulator [12]. Both lossy compression algorithms cause an increase in the simulated run time.

The absolute increase in the simulated run time for both algorithms is quiet similar as can be seen in Figure 5. Another 


\begin{tabular}{|l|c|c|c|c|}
\hline & $\begin{array}{c}\text { ITFComp } \\
\text { (periodicity-raw) }\end{array}$ & $\begin{array}{c}\text { ITFComp } \\
\text { (periodicity-SEQUITUR) }\end{array}$ & SC.I & SC.I+Ntup \\
\hline cjpeg & 0.00318 & 0.00198 & 0.0208 & 0.0068 \\
\hline mad & 0.00181 & 0.00048 & 0.0123 & 0.00562 \\
\hline sha & 0.00190 & 0.0011 & 0.0144 & 0.0023 \\
\hline blowfish_e & 0.00175 & 0.0000625 & 0.0391 & 0.0102 \\
\hline
\end{tabular}

Table 3: The average compression ratio achieved by ITFComp compared with the stream cache algorithm.

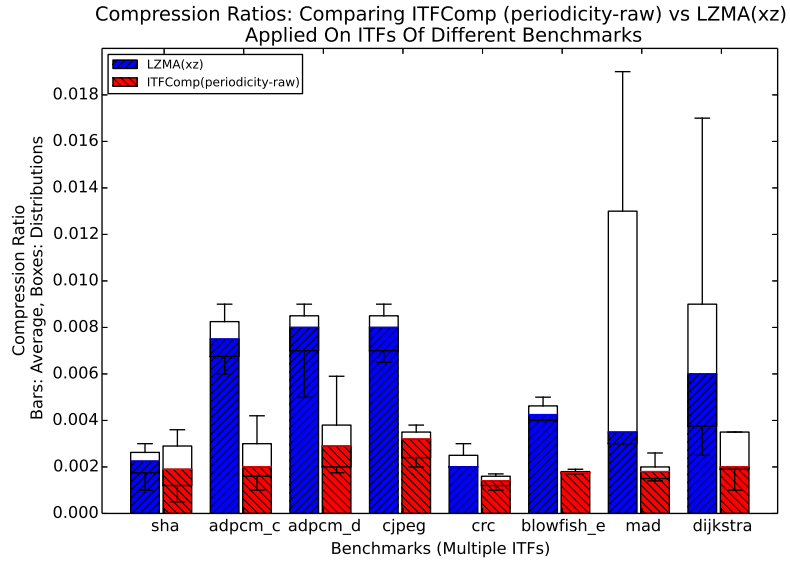

Figure 4: The compression ratio achieved by ITFComp combined with LZMA, compared to the general purpose compression algorithm $\operatorname{LZMA}(\mathrm{xz})$.

thing to notice is that the increase in the simulated run time is larger, the larger the simulated run time of the input ITF is. This can be explained by being more prone to lossy data memory addresses and cache misses in the decompressed ITF during a longer simulation.

\subsection{Wall Clock Simulation Time}

This is a measure of the decompression time of the ITFs during their simulation. ITFComp and $\operatorname{LZMA}(\mathrm{xz})$ compressed ITFs were decompressed and streamed into the used simulator. The wall clock simulation time was measured. The process was repeated 20 times for 3 different ITFs from 3 different benchmarks with varying sizes.

The results in Figure 6 show that on average, the decompression and simulation time of ITFs for both compression methods is similar, with a slight increase for ITFComp. Therefore, ITFComp introduces a small overhead on the decompression process, but the ratio of that increase is negligible.

In summary, the three used metrics shows that the suggested compression method manages to reduce the size of ITFs on evaluation set more than general purpose and domain specific compression methods. It also shows that despite being lossy in some modes, the resulting compressed ITFs manages to keep their performance characteristics. However, there is a slight increase on the decompression time of the ITFs that can be ignored compared with the large simulation time.

\section{SUMMARY AND FUTURE WORK}

Emulating hardware components during a new hardware design is used to reduce its design costs in terms of money and

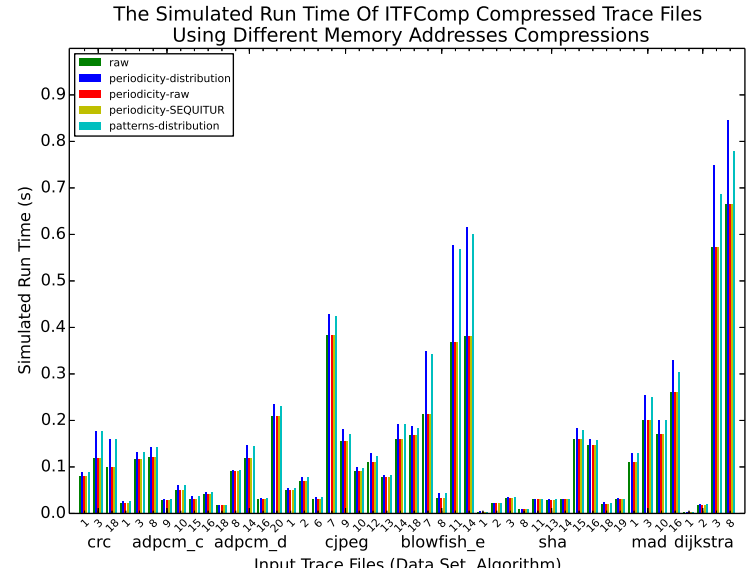

Figure 5: The simulated run time of compressed ITFs using different data memory addresses compression techniques in ITFComp.

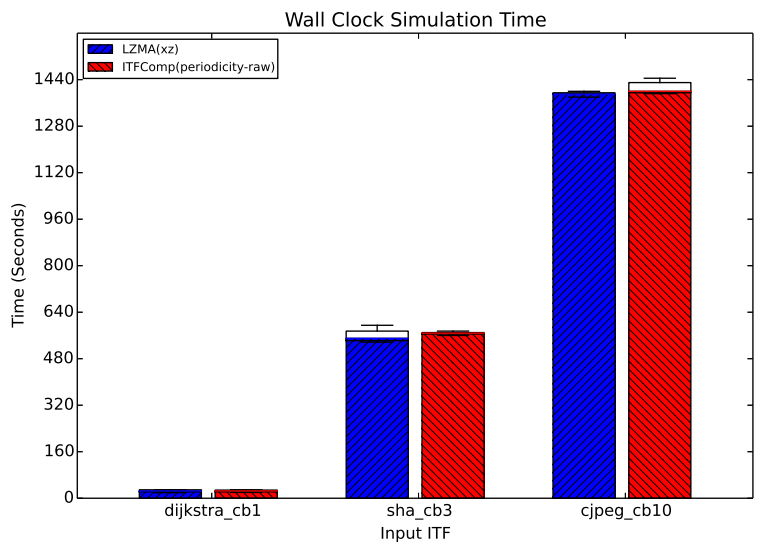

Figure 6: The wall clock simulation time of three compressed ITFs using $\operatorname{LZMA}(x z)$ and ITFComp. The bar plots represent the average, while the boxes represent the distribution.

time. Hardware simulations use Instruction Trace Files (ITF) as an input, generated from tracing benchmarks and programs executed on similar hardware. ITFs tend to be large in size as with modern processors, billions of instructions are executed per second. This causes a storage challenge.

Compression is used to address this challenge. However, general purpose compression algorithms lack domain specific knowledge, represented in the structure of used ITFs. Such 
knowledge can be used to further reduce ITFs size when applying general purpose compression to better deal with the storage challenge.

In this work, we present ITFComp; a new algorithm that deals with ARM architecture ITFs, as ARM architecture is used in most modern smartphones and hand held computational devices. ITFComp works by structuring an ITF into three components: a sequence of executed basic blocks, a template for each basic block, and data memory addresses accessed by instructions within the ITF.

The list includes any special execution flags of the basic blocks, as well as information about interrupts. Templates are extracted from an instance of a basic block with all flags and data memory addresses replaced by place holders. Data memory addresses are compressed in a way that allows for random access and use information about instructions access behaviors such as locality and patterns.

ITFComp helps to improve general purpose compression methods on input files up to four times when compared to LZMA, and 10 times better when compared to DEFLATE. ITFComp achieves better compression ratios when compared to the domain specific cache streams compression method.

Future work to enhance ITFComp would include finding patterns within the basic blocks list as there is usually locality within executed basic blocks. This locality is a result of high level language structures such as conditional basic blocks following a loop body. More compression methods could be tested for data memory addresses. For example, a distributions that pay more attention to the samples order using heuristics. The list of basic blocks could also be used to partially decompress an ITF generating certain parts of interest for a specific simulation.

\section{REFERENCES}

[1] C.W.E.N. CHEN, C.J. KU, and T.J.Y.E. LIU. Efficient trace file compression design with locality and address difference.

[2] L. Peter Deutsch. Deflate compressed data format specification version 1.3, 1996. RFC 1951 - Network Working Group.

[3] Davy Genbrugge, Lieven Eeckhout, and Koen de Bosschere. Accurate Memory Data Flow Modeling in Statistical Simulation. In 20th annual international conference on Supercomputing, pages 87-96, June 2006.

[4] Craig G.Nevill-Manning and lan H. Witten. Identifying hierarchical structure in sequences: A linear-time algorithm. Journal of Artificial Intelligence Research, 7:67-82, 1997.

[5] M. R. Guthaus, J. S. Ringenberg, D. Ernst, T. M. Austin, T. Mudge, and R. B. Brown. Mibench: A free, commercially representative embedded benchmark suite. In Proceedings of the Workload Characterization, 2001. WWC-4. 2001 IEEE International Workshop, WWC '01, pages 3-14, Washington, DC, USA, 2001. IEEE Computer Society.

[6] E.E. Johnson and Jiheng Ha. Pdats lossless address trace compression for reducing file size and access time. In Computers and Communications, 1994., IEEE 13th Annual International Phoenix Conference on, page 213, apr 1994.

[7] E.E. Johnson, Jiheng $\mathrm{Ha}$, and M. Baqar Zaidi. Lossless trace compression. Computers, IEEE Transactions on, 50(2):158 -173, feb 2001.
[8] N. Malik, R.J. Eickemeyer, and S. Vassiliadis. Architectural effects on dual instruction issue with interlock collapsing alus. In Computers and Communications, 1993., Twelfth Annual International Phoenix Conference on, pages 42 -48, mar 1993.

[9] A. Milenkovic and M. Milenkovic. Exploiting streams in instruction and data address trace compression. In Workload Characterization, 2003. WWC-6. 2003 IEEE International Workshop on, pages 99-107, Oct 2003.

[10] M. Milenkovic, A. Milenkovic, and M. Burtscher. Algorithms and hardware structures for unobtrusive real-time compression of instruction and data address traces. In Data Compression Conference, 2007. DCC '07, pages 283-292, March 2007.

[11] ARM Holdings plc. Annual report 2013: Strategic report. Technical report, 2013.

[12] L. Pustina, S. Schwarzer, P. Martini, J. Muurinen, and A. Salomaki. A methodology for performance predictions of future arm systems modelled in uml. In Systems Conference, 2008 2nd Annual IEEE, pages 1-8, April 2008.

[13] A. Dain Samples. Mache: No-loss trace compaction. Technical Report UCB/CSD-88-446, EECS Department, University of California, Berkeley, Sep 1988.

[14] J. Seward. bzip2 and libbzip2. avaliable at http://www. bzip. org, 1996.

[15] A.J. Smith. Two methods for the efficient analysis of memory address trace data. Software Engineering, IEEE Transactions on, SE-3(1):94 - 101, jan. 1977.

[16] Wikipedia. LempelâĂȘzivâĂȘmarkov chain algorithm wikipedia, the free encyclopedia, 2012. [Online; accessed 02-November-2012]. 